\title{
HISTÓRICO, PERSONAL Y POLÍTICO: Psicología y Revolución ${ }^{42}$
}

\author{
Ian Parker ${ }^{43}$
}

El desarrollo de la psicología se ha visto interrumpido, a menudo, por eventos históricos que escapan a su control, los que hasta el día de hoy marcan esta disciplina. En vez de tratar las revoluciones simplemente como vestigios históricos de tiempos pasados, podemos considerarlas como elementos claves en la memoria colectiva de la psicología y examinarlas como parte de la "historia del presente". La historia convencional de la psicología es una versión parcial, elaborada desde un punto de vista particular, y la forma en que nosotros la volvemos a relatar, desde la perspectiva de los oprimidos, tiene consecuencias para cómo pensamos en nosotros mismos. Este capítulo trata de las revoluciones que obligaron a los psicólogos a repensar su quehacer, y sobre cómo las ideas radicales que en algún momento afectaron la disciplina perviven en la actualidad.

\section{Las críticas internas del ámbito de la psicología reflejan los debates dominantes}

Cada uno de los choques revolucionarios que ha sostenido la disciplina y que describiremos en este capítulo, tuvo el efecto de dividir la compartimentalización de la disciplina en áreas de estudio separadas. Dichas áreas, como por ejemplo la psicología del desarrollo, la psicología del lenguaje, la psicología social y la psicología psicoanalítica, a menudo se tratan como si hubiese divisiones herméticas entre ellas. La psicología de la mujer, la psicología biológica, la psicología cognitiva y las teorías de la conciencia están separadas en especializaciones que compiten entre sí. Incluso las vinculaciones establecidas en las investigaciones "interdisciplinarias" suelen servir para reforzar las miradas ideológicas dominantes de lo que son las personas.

42 Publicado en Revolution in Psychology. Alienation to emancipation, London: Pluto Press. Traducción y publicación autorizadas por el autor.

43 Profesor de la División de Psicología y Cambio Social, Manchester Metropolitan University. 
Asimismo, los desafíos a la ideología dominante que ocurrieron durante las revoluciones, necesariamente han sido, también, desafíos para la psicología. Una revolución remece las categorías que utilizamos para darle sentido a la experiencia; muestra cuán artificial y -a la vez- cuán convincente es la separación entre lo individual y lo social bajo el capitalismo, y la actividad de cambiar y al mismo tiempo interpretar el mundo revela que nuestra individualidad es social de cabo a rabo. El desafío a la psicología alienante, que separa a las personas, unas de otras, y nuestro sentido de nosotros mismos de nuestras capacidades creativas, también implica un desafío a la imagen de la mente y el comportamiento como compartimentalizados en áreas separadas de estudio. La simple investigación "interdisciplinaria” que intenta parchar estos componentes separados ya no es suficiente; como tampoco lo es la investigación "interdisciplinaria" que respeta la existencia de subdivisiones académicas en la psicología. ${ }^{44}$

\section{Las alternativas se manifiestan al surgir crisis políticas que obligan a los psicólogos a cambiar}

Las revoluciones ponen patas arriba las sabidurías recibidas, no obstante una revolución no es sólo una época de trastornos caóticos. No es una abolición nihilista de todo lo que pasó antes, y esa imagen equivocada de revolución es una suerte de garantía ideológica para mantener la ley y el orden tal como son en la actualidad, de manera que los privilegiados y poderosos sean quienes defiendan a la civilización de la barbarie. De hecho, esta forma de ver la revolución -como un quiebre irracional de la vida civilizada donde la turba se impone sobre la racional toma de decisiones- es una manera profundamente "psicológica" de ver el cambio. ${ }^{45}$ Es una imagen del cambio cargada con temor a lo que ocurrirá si las personas toman las riendas de sus propias vidas.

Una revolución es una oportunidad para descubrir nuevas formas de vivir, de resaltar aspectos de la creatividad y la esperanza humanas que por lo común son reprimidos. Los logros artísticos y científicos, así como las condiciones materiales en las que es posible superar la separación entre el "trabajo" y el "ocio", son transformados y ampliados por la revolución, no eliminados. Al mismo tiempo

44 Curt (1994) intentó interrumpir la forma en que operan las investigaciones "interdisciplinarias", a fin de cosificar cada componente disciplinario en su llamamiento por la investigación "transdisciplinaria" en la psicología social; su libro es una extraña mezcla de construccionismo social e investigación cuantitativa escrito por una colectividad cuyo nombre, "Beryl Curt", fue diseńado para parodiar y subvertir la lealtad de la psicología británica para con el trabajo de Cyril Burt.

45 El trabajo de Le Bon (1986) sobre la multitud es el texto más influyente y fue seguido por varios estudios psicológicos sobre los peligros de los instintos de "la manada" y de la "mente grupal” (Véase, p. ej., Trotter, 1919; McDougall, 1927). 
que los sistemas existentes de conocimiento son reevaluados, la revolución es una oportunidad para hacer un recuento de lo que puede conservarse de las viejas maneras de comprender el mundo. Es en aquellos momentos cuando las disciplinas que hacen una virtud del aislamiento individual, como la psicología, se sacuden hasta sus bases, y podemos empezar a vislumbrar el surgimiento de algo completamente distinto. ${ }^{46}$

\section{Un nuevo enfoque sobre la actividad se presentó en la Revolución rusa de 1917}

La Revolución de octubre de 1917 fue un evento bastante inesperado para muchos marxistas tradicionales (especialmente los que trataban el marxismo como una ocupación académica o una visión del mundo cuasi religiosa), así como para la clase gobernante. Aquellos marxistas que sostenían que los regímenes feudales serían reemplazados por regímenes capitalistas antes de que hubiese alguna posibilidad de una revolución socialista, vieron esas "etapas" ostensibles de la historia, comprimidas y pasadas por alto. Concepciones alternativas de "desarrollo combinado y disparejo" de los centros capitalistas y de la periferia "subdesarrollada" ahora podían comprender un poco mejor lo sucedido en Rusia. Dichas concepciones alternativas, enraizadas en la praxis de la cambiante sociedad rusa, mostraron que sería imposible desarrollar la revolución por sí sola, como el "socialismo en un único país". ${ }^{47}$ Los cambios revolucionarios incluyeron innovaciones en el arte, la ciencia y la vida privada, con la revocación de leyes relativas al matrimonio, la sexualidad y la infancia, y el reconocimiento de los derechos de los oprimidos bajo el antiguo régimen. Los cambios revolucionarios acontecidos desde 1917 hasta principios de 1920 fueron de gran alcance, sin embargo, el aislamiento del nuevo régimen y la cristalización de la burocracia bajo Stalin derivaron en la represión de quienes no acataban las normas partidarias. En el ámbito de la vida personal, también se revirtieron muchos cambios progresivos en la situación posicional de la mujer, por ejemplo, y Stalin reforzó su poder apelando una vez más a los vetustos valores reaccionarios de "la nación" y "la familia". ${ }^{48}$

46 Los intentos marxistas en la psicología de abordar la naturaleza de "la vida cotidiana" han tratado el impacto de la rutina y de la revolución sobre la consciencia, y por lo general su opinión de las teorías psicológicas convencionales es que carecen de utilidad (Véase, p. ej., Hayes, 1996; Hogget, 1996). Para consultar un buen trabajo reciente sobre este tema, que recurre más extensamente a la teoría social y la acción política como puntos de referencia, véase Bratsis (2006) y Stephenson y Papadopoulos (2006).

47 Para una historia de la revolución escrita por una persona informada que trata estos puntos, véase Trotsky (1977), y para un análisis del ascenso de la burocracia, véase Trotsky (1973).

48 Véase, por ejemplo, el relato de Colletti (1970) sobre la restauración de la vieja ideología zarista de nación y familia bajo el gobierno de Stalin; véase también Zaretsky (1976) para 


\section{La investigación de Vygotsky en Rusia llevó a nuevas nociones de desarrollo y lenguaje}

La revolución fue el contexto para las nuevas ideas sobre la infancia que desafiaron las ideas fijas sobre las edades y etapas. La revolución confundió a aquellos que esperaban que la historia se desarrollara mediante una secuencia ordenada, y fue así que los psicólogos del desarrollo fueron también sensibilizados ante la posibilidad de repentinos cambios cualitativos a medida que los niños comenzaban a adquirir el habla. El lenguaje en sí fue reexaminado, no meramente como un medio para comunicar ideas desde la cabeza de una persona a la de otra, sino como un proceso social colectivo mediante el cual las personas pueden pensar en sí mismas y en los demás. Nuestro sentido de individualidad, y nuestra "psicología" separada, son consecuencia de ciertas formas del lenguaje, y de ciertas formas de hablarlo. ${ }^{49}$

Podemos apreciar un contraste entre las teorías psicológicas predominantes del desarrollo, que aún son populares en los textos escolares en EEUU y Europa, basadas en la obra del psicólogo suizo Jean Piaget, y el trabajo del psicólogo ruso Lev Vygotsky. Piaget visualizó cómo el desarrollo cognitivo interno conduce al nińo hasta el punto donde aprendería el lenguaje, y pasaría desde el pensamiento individual egocéntrico a las relaciones sociales con otros. Vygotsky, en cambio, mostró cómo el pensamiento se desvinculaba del lenguaje, y cómo el individuo, asimismo, quedaba desvinculado de la actividad colectiva, aunque todavía dependiente de ella. ${ }^{50}$ Una teoría del lenguaje se fusionó con una teoría del desarrollo para dar origen a una imagen bastante novedosa del ser humano como un ser social de punta a cabo; fue una psicología revolucionaria. ${ }^{51}$

\section{El desarrollo de la teoría de la actividad intentó redefinir la psicología}

Las ideas de Vygotsky no eran idiosincrásicas, ni libres de contexto, no más de lo que el pensamiento de un individuo puede estar separado de las personas de

una discusión de sus implicancias para las políticas marxista y feminista.

49 Véase Friedman (1990) sobre la Unión Soviética de 1920 como trasfondo para el desarrollo de las ideas de Vygotsky.

50 Véase Vygotsky (1962) para una traducción algo truncada de uno de sus trabajos claves para el público norteamericano. Bickley (1977) discurre sobre el trabajo de Vygotsky como una psicología "dialéctica materialista”. Walkerdine (1982) revisa y desarrolla esta diferencia entre Piaget y Vygotsky.

51 Para mayores antecedentes sobre el argumento de que se trataba de una "psicología revolucionaria” véase Newman y Holzman (1993); véase también Holzman (1996) sobre cómo el trabajo de Vygotsky debería ser "completado" (por Newman). Para una exposición clásica sobre esta particular alternativa marxista-vygotskiana en la psicología, véase Hood y Newman (1983), y para un relato más actual sobre el rumbo del trabajo de Newman, véase Holzman y Mendez (2003). 
su entorno. Sus ideas formaban parte de debates sobre la naturaleza del lenguaje, que lo trataban como un sistema "semiótico" compartido de significados en el que cada elemento está definido según su relación con los demás elementos y por la lucha entre las clases sociales y otras categorías sociales. ${ }^{52}$ Una amplia redefinición de la relación entre el lenguaje y el desarrollo estaba siendo elaborada en ese momento por un conjunto de enfoques a menudo agrupados bajo la etiqueta de la teoría de la actividad..$^{53}$

En vez de centrarse en objetos que parecían estar separados por haber sido "reificados", tan a fondo convertidos en cosas, las complejas relaciones dialécticas entre las cosas se volvieron el foco de estudio. Esto brinda una manera de pensar en la alienación, como la separación del pensamiento del lenguaje y como una consecuencia de tratar cosas tales como la inteligencia y la cognición como medibles, para luego definir las características de la propia identidad individual. Vygotsky murió en 1934, poco después de que el terror estalinista alcanzara su auge, y entonces los teóricos de la actividad tuvieron que adaptarse y ajustar su trabajo a fin de evadir la atención desmedida de la burocracia. ${ }^{54}$

\section{El trabajo de los marxistas en Francia redefinió la personalidad en relación con el trabajo}

La existencia de la Unión Soviética dio confianza a los movimientos de liberación que luchaban por su independencia de las potencias imperialistas de occidente; esto sucedía al mismísimo tiempo que la burocracia estalinista intentaba ejercer su propio gobierno sobre aquellos que tácticamente apoyaba. La existencia de fuertes partidos comunistas pro-soviéticos en distintas partes del mundo también tenía su pro y su contra para activistas y académicos de izquierda. La relación entre las potencias imperialistas y las viejas colonias fue cambiada por la existencia de la Unión Soviética, la que, pese a todos los esfuerzos de la burocracia por mantener las cosas bajo control, dio esperanza a los que luchaban contra el colonialismo. El trabajo del psiquiatra radical Frantz Fanon es un ejemplo claro. Originario de Martinica, trabajó en Argelia junto al movimiento de liberación contra la ocupación francesa y escribió sobre los efectos de la colonización sobre

52 El revolucionario trabajo sobre el lenguaje de Valentín Volosinov (1973) es analizado por Greenslade (1996).

53 Véase Holzman (2006) para una recopilación de artículos que pasan revista al estatus actual de las perspectivas vysgotskiana y de la teoría de la actividad.

54 Para un relato de bastante buen nivel de las ideas de Vygotsky, véase Kozulin (1989), y para un tratamiento extenso (si bien a ratos bastante reaccionario) del trabajo de Vygotsky, véase Kozulin (1994). 
lo que pasó a ser el mundo interno del colonizado y el colonizador ${ }^{55}$. En Francia, el partido comunista giraba en torno a las órdenes de la burocracia soviética y efectuaba sus propias purgas y cazas de brujas de los disidentes; pero al mismo tiempo brindó un espacio para transmitir algunas de las innovaciones desde la Rusia revolucionaria y para elaborarlas en relación a la teoría marxista. El trabajo de Lucien Sève sobre la redefinición de la personalidad es un ejemplo clave. ${ }^{56}$ Sève construyó, sobre la comprensión marxista, de que no hay esencia del ser humano que la psicología puede capturar y definir para todos los tiempos, sino que el ser humano es "un ensamble de las relaciones sociales". ${ }^{57}$

De este modo, el trabajo de los teóricos rusos de la actividad fue dirigido a entender cómo la personalidad se vinculaba al trabajo y cómo la vida creativa del obrero quedaba distorsionada por el tiempo que, en una sociedad capitalista, tenía que vender al empleador. ${ }^{58}$

\section{La "psicología crítica" alemana redefinió la actividad y luego la "memoria"}

En Alemania occidental el partido comunista pro-soviético se encontraba en una situación mucho más difícil que su contraparte francesa tras el fin de la Segunda Guerra Mundial. Sin embargo, mientras que a menudo los comunistas del oeste eran obligados a pasar a la clandestinidad -con los ultra izquierdistas embarcándose en desastrosas actividades individuales terroristas para intentar revolver las cosas- la proximidad de Alemania oriental les otorgó de hecho a algunos radicales cierto consuelo intelectual. De nuevo, podemos ver el rol paradójico y contradictorio del bloque soviético como condición de posibilidad y obstáculo para los movimientos de izquierda.

Las revoluciones estudiantiles de 1960 inspiraron a un psicólogo, hasta ese entonces bastante tradicional, Klaus Holzkamp, en Berlín occidental, a basarse en y redefinir la teoría de la actividad ${ }^{59}$. Un importante movimiento alternativo

55 Véase Fanon $(1967,1970)$. Heartfield (2002) proporciona el interesante argumento de que la lucha argelina fue crucial para el desarrollo del existencialismo y luego de la teoría postestructuralista en Francia.

56 Sève (1978); para una explicación del trabajo de Sève, véase Shames (1981); para una explicación más crítica sobre el lugar de Sève en el contexto del Partido comunista francés, véase O’Donnell (1982).

57 Marx utiliza esta frase en su "Tesis sobre Feuerbach".

58 Véase Leonard (1984) para un intento de aplicar el trabajo de Sève en el contexto del trabajo social británico.

59 Tolman (1994) provee una excelente introducción al trabajo de Holzkamp y al trasfondo político del desarrollo de la psicología crítica alemana; para una explicación de esta línea de la psicología crítica ahora en Alemania, véase Held (2006); y en Austria véase Sanin (2006). 
de izquierda, conocido como Kritische Psychologie, se fue desarrollando, lo cual le proporcionó un público al trabajo de Holzkamp sobre una nueva ciencia del sujeto marxista, enfocada sobre la potencia para la acción ${ }^{60}$. Este trabajo fue avanzado por la marxista-feminista Frigga Haug mediante una metodología colectiva grupal de trabajo de memoria. Esta noción de memoria colectiva dista bastante de los estudios sobre la memoria individual en la psicología. ${ }^{61}$

\section{Un nuevo enfoque sobre el poder surgió tras los hechos de 1968 en Francia}

Los eventos de mayo en París, las rebeliones estudiantiles y obreras que se repitieron en todo el mundo a fines de 1960, fueron una sorpresa tanto para la izquierda como para la clase gobernante francesa. Algunas de las chispas iniciales que prendieron la revuelta estudiantil -protestas en contra de la segregación de los sexos en los dormitorios residenciales- ya señalaban cuán importante era el vínculo entre la vigilancia de la vida privada y las grandes cuestiones políticas de poder estatal. A medida que la rebelión estudiantil se difundía, se establecieron vínculos con huelgas en fábricas automotoras en las afueras de Paris y en otras zonas de Francia. Este movimiento revolucionario fue en muchos sentidos un fenómeno de izquierda, que se unía a las luchas en torno a la sexualidad y la orientación sexual, contra el racismo y las formas simbólicas de poder. Los "situacionistas", por ejemplo, se proponían alterar la "sociedad del espectáculo" que embrujaba y tranquilizaba a las personas; y los lemas del movimiento estudiantil reflejaban su visión romántica de algo radical que se abría camino con fuerza en contra de la represión personal y estatal. ${ }^{62}$ Mientras arrancaban los adoquines de las calles de París y los arrojaban a la policía, realmente parecía que el eslogan: "bajo las baldosas, la playa" pudiese ser verdad. Luego de que la ley y el orden se hubieran restaurado, sin embargo, la cínica respuesta de los reaccionarios "nuevos filósofos" fue que, dada la naturaleza humana, los estudiantes debían haber reconocido la dura verdad de un caso de "bajo la playa, las baldosas". La psicología, desafiada por los eventos, también volvía al ruedo.

60 La colección de Tolman y Maiers (1991) es la guía más útil para el ámbito de trabajo en esta tradición en inglés; para un breve resumen véase Maiers y Tolman (1996), y Holzkamp (1992).

${ }^{61}$ Véase Haug $(1987,2000)$ para ejemplos y una discusión teórica sobre el "trabajo de memoria”.

62 Véase Debord (1977) para un análisis de la sociedad del espectáculo, y Plant (1993) para una historia y análisis del impacto de Debord y el situacionismo. 


\section{El trabajo de Foucault sobre la locura trazó la división entre la razón y la irracionalidad}

Algunos miembros del partido comunista francés, y particularmente su rama juvenil, apoyaron las revueltas, pero la cúpula quería desviar una revolución que escapaba a su control y evitar cuestionamientos de la autoridad del partido. Durante los eventos de 1968, Michel Foucault, que se había formado como psicólogo clínico y realizado trabajo forense en las cárceles durante la década de $1950,{ }^{63}$ se desempeñaba como docente en Túnez, aunque ya estaba involucrado en los acontecimientos allí, albergando a estudiantes que protestaban contra el régimen. ${ }^{64}$ Foucault se había salido del partido comunista en 1953, en parte debido el antisemitismo de los partidos comunistas estalinizados inmediatamente después de la muerte de Stalin, y en parte debido a la homofobia estalinista. ${ }^{65}$ Lo político y lo personal ya se entrelazaban en sus ideas políticas y, luego, en su trabajo teórico.

El trabajo de Foucault sobre la historia de la locura y cómo la civilización moderna separa la razón de aquellos aspectos de la experiencia humana que le resultan insoportables y perturbadores, aún estaba en deuda con el marxismo. ${ }^{66}$ Su trabajo mostró cómo la locura y lo irracional se cosificaban, convertidos en un inconsciente para ser descifrado por los psicoanalistas; de esta manera Foucault entregó una alternativa a las versiones del psicoanálisis que simplemente se enfocaban en lo que estaba reprimido al interior de cada individuo. ${ }^{67}$ Pese a que su trabajo ha sido citado por el movimiento anti-psiquiatría, Foucault no romantizó la locura como si ésta fuera una experiencia esencialmente verdadera que revienta ante las ansias de liberarse de las cadenas de la razón. ${ }^{68}$

\section{El involucramiento con las luchas carcelarias llevaron a nuevos trabajos sobre la disciplina y el castigo}

Llegó el verdadero punto crucial cuando Foucault se involucró en las rebeliones de algunos movimientos emergidos de los hechos de 1968. Los prisioneros,

63 Véase Parker (1995b) para un breve recuento de los antecedentes de Foucault en la psicología.

64 Véase la biografía intelectual de Macey (1994) para un testimonio sobre el tiempo que pasó Foucault en Túnez.

65 Véase Macey (1994) para una exposición del tema.

${ }_{66}$ En una serie de entrevistas Foucault (1991) reflexiona sobre la influencia ejercida por Marx sobre su obra.

67 Miller (1989) entrega una evaluación (psicoanalítica) de la compleja relación de Foucault con el psicoanálisis; para una buena crítica marxista del psicoanálisis véase Timpanaro (1976).

68 Deleuze y Cuattari (1977) se aproximaron bastante a este tipo de romanticización de la esquizofrenia, principalmente como una reacción a formas del psicoanálisis que parecían demonizarla. 
organizados para protestar en contra de las condiciones brutalmente deshumanizantes, adquirieron voz gracias a grupos como Group for Information on Prisons (Grupo por la Información sobre Prisiones), del que Foucault formaba parte. Este involucramiento práctico lo llevó a escribir un libro que rompería esquemas: Discipline and Punish (Vigilar y Castigar), en que mostraba cómo las prácticas de vigilancia análogas a las carcelarias habían surgido en la sociedad moderna desde principios del siglo XIX. ${ }^{69}$

El monitoreo y la grabación de cada individuo formaban parte de un aparato de control en que la gente comenzó a desarrollar una sensación de sí mismos como en constante observación, incluso cuando no lo estaban. Una disciplina clave en este aparato de individualización y control fue precisadamente aquella en la que Foucault fue entrenado en primer lugar: la psicología. La concepción de poder de Foucault, como algo que mantenía a las personas en relaciones de sumisión frente a la autoridad, y no como una fuerza que algunos individuos ejercen deliberadamente sobre otros, era todavía bastante marxista. El mismo Marx no trató de explicar por qué ciertos capitalistas explotaban malvadamente a pobres obreros; esa clase de explicación psicológica fue rechazada en favor de una explicación político-económica de cómo las personas adquirieron consciencia de quiénes eran, en el contexto de ciertas relaciones de poder históricamente específicas. Es precisamente por eso que necesitan interpretar al mundo si lo quieren cambiar.

\section{Estudios sobre la sexualidad llevaron a relatos de la confesión como vinculada al poder}

Foucault señaló cómo una explicación psicológica era parte del problema, cómo enturbiaba la visión de lo que estaba realmente en juego. Luego, su análisis histórico de la "sexualidad" se dirigió a las cosas que parecían estar reprimidas por el poder. La gente llegó a creer que algo había de genuino y liberador por debajo de la superficie -en tiempos de revolución parecía como si fuera verdadero eso de que "bajo las calles pavimentadas, la playa"-. Este análisis arroja nuevas luces sobre la asignación de hombres y mujeres biológicos a sus cuerpos sexuales distintos, históricamente construidos, y por consiguiente sobre toda la preocupación por las diferencias de género en la psicología. También plantea interrogantes de orientación sexual y avanza más allá de las categorías de heterosexualidad y homosexualidad como caminos de vida separados.

Lo seductor de esta atrayente noción condujo a la gente hacia formas de terapia en donde pensaban que, si hablaban libremente del tema, algo se liberaría

69 Véase Foucault (1977) para un análisis de la vigilancia en la sociedad capitalista del siglo XIX. 
y serían más felices y saludables. Foucault mostró cómo la disciplina en la psicología era acompañada por la confesión, como el rostro empático del profesional que invita a alguien a contarle todo y le persuade que se sentirá mejor por haberlo hecho.

\section{Una nueva noción de la subjetividad como constituida históricamente desafió a la psicología}

El vínculo entre la disciplina y la confesión ha brindado un nuevo modo de pensar en la actividad de los psicólogos -ellos forman parte del sistema, les guste o no, y más aún cuando intentan relacionarse amablemente con él-y también sobre la psicología en sí. Foucault mostró cómo era posible desarrollar una noción completamente distinta del sujeto individual y de nuestra comprensión reflexiva sobre nuestra propia subjetividad. Su trabajo es a veces caricaturizado como conllevando la muerte del sujeto, ${ }^{70}$ pero lo que él produjo fue una "historia del presente" como una historia que comienza por la forma cómo somos, y que muestra cómo se ha llegado a ser de tal modo.

Ese trabajo ha sido considerado como negativo por algunos marxistas, quienes ven en él una amenaza a su propio poder y liderazgo, pero ha sido una inspiración para marxistas críticos, y otros que buscan un mejor entendimiento del proceso revolucionario. Su trabajo es un golpe particularmente duro contra la tradición estalinista del marxismo, y genera atención sobre el impacto de esa tradición en la forma en que grupos maoístas o trotskistas han alegado lealtad al marxismo revolucionario y sus organizaciones. ${ }^{71}$ Con todos los problemas en el trabajo de Foucault, este aún provee un recurso conceptual para re-energizar genuinas políticas revolucionarias, y muestra cómo el destino de la subjetividad individual se encuentra enlazada con el proceso social. ${ }^{72}$

70 Para este argumento véase la conclusión de Foucault (1970) en The Order of Things (El orden de las cosas); es la "figura" del hombre como punto de referencia conceptual para las explicaciones de cómo se produce el conocimiento que [Foucault] cuestiona, no a los seres humanos individuales; y los críticos a menudo parecen deliberada y obtusamente pasar por alto esta diferencia.

71 Causa poca sorpresa el que fue desde dentro del estalinismo que quienes se sintieron desilusionados con lo que percibían como una política "revolucionaria" vetusta y autoritaria, se dirigieron erróneamente al "Eurocomunismo" reformista social demócrata como si fuera una alternativa. Dada su experiencia de lo que era la política partidista marxista, era entendible que concluyeran que el único camino hacia adelante era adoptar una forma de postmarxismo (para un ejemplo véase Laclau y Mouffe, 2001). Para un análisis del impacto de la falta de democracia al interior de la tradición estalinista, junto con la lógica centrífuga del "socialismo en un solo país", véase Mandel (1978).

72 Véase Sawaki (1991) para una crítica feminista de Foucault. 


\section{Un nuevo enfoque sobre la Naturaleza aparece desde 1970 Segunda Ola Feminista en Estado Unidos}

En Estados Unidos la temprana actividad comunista de los trabajadores en busca de una alternativa al capitalismo fue mucho más rápida y fácilmente erradicada que en Europa. La combinación de un partido comunista local fuertemente organizado y leal a la Unión Soviética, y la caza de brujas de McCarthy en contra de los implicados en actividades "anti-americanas" después de la Segunda Guerra Mundial, hizo la vida muy difícil para los marxistas. Los psicólogos que se identificaban como marxistas tuvieron que ser muy cuidadosos, y hubiese parecido estrafalario intentar desarrollar allí una "psicología marxista" ${ }^{73}$ Las expresiones de descontento con el capitalismo tomaron diferentes formas y hubo varios movimientos que construyeron una crítica del estatus quo enfocada sobre la opresión racial o la explotación sexual. Las protestas antirracistas encontraron cabida en trabajos académicos; los que fueron hasta cierto punto asimilados en la psicología, y hoy por hoy inspiran la teoría postcolonial, de la que los radicales en psicología aún podrían aprender. Sin embargo, han sido las feministas socialistas de la "segunda ola" 74 quienes han abordado las conexiones entre vida privada y lucha política, y sus trabajos remecieron a la psicología. Los análisis del sistema capitalista como entramado con, y reforzado por el patriarcado, como un sistema de opresión, en que los hombres dominan a las mujeres y los hombres mayores a los jóvenes, resultaron muy perturbadores para los psicólogos varones. Actualmente la crítica se ha amainado y el capitalismo ha encontrado formas de incorporar a las mujeres como gerentes, y encubrir lo que era más radical en el feminismo con la ideología del post-feminismo. ${ }^{75}$

\section{Estudios feministas sobre la primatología levantaron interrogantes acerca de la construcción de la naturaleza}

La lectura acabada de las "investigaciones sobre los primates" demostró que nuestras ideas acerca de la naturaleza en la psicología ostensiblemente derivan

73 Véase Harris (1990) para un estudio de la psicología en relación a la izquierda en EEUU, y Harris (1995) para un análisis de la relación histórica entre la psicología y el marxismo en EEUU.

74 Las luchas de la "primera ola" tuvieron lugar a comienzos del siglo [XX], principalmente en torno al tema del derecho a voto; la "segunda ola" fue movilizada por las revueltas de los ańos sesenta, y la "tercera ola" con el cambio al siglo XXI. Véase Rowbotham (1973) para las historias de las primeras dos olas, y Zavos (2005) para revisar material sobre la "tercera ola" (particularmente en relación al activismo feminista en torno a la psicología).

75 Véase Gamble (2001) para los debates al interior del feminismo y Eisenstein (1996) para un relato de lo que pudiese significar en la práctica (con un enfoque sobre el fenómeno "femócrata" en Australia). 
de estudios sobre el comportamiento primate, cuando dichos estudios en realidad proyectan en el comportamiento de los simios nuestras suposiciones ideológicas específicas a nuestra cultura. ${ }^{76}$ Los estudios sobre el apego en los simios bebés separados de sus madres y luego obligados a relacionarse con madres sustitutas de malla de alambre son parte importante del trabajo de Donna Haraway. El control masculino de la reproducción está estructurado no sólo en las descripciones que el investigador Harry Harlow brinda sobre el comportamiento primitivo, sino además, en la misma forma en que se armaron los estudios. Por ejemplo, las hembras primates aisladas eran incapaces de concebir debido a las condiciones peculiares a las que ya habían sido sometidas. Se las inseminaba artificialmente mediante el uso de lo que Harlow y sus colegas llamaban el "rack de la violación". ${ }^{7}$

Haraway demostró cómo las fantasías masculinas de controlar la naturaleza femenina se reprodujeron en los estudios de monos huérfanos para hacer parecer que Harlow hubiera descubierto dicha naturaleza, cuando en realidad él la construía. Esta es una segunda naturaleza que funciona ideológicamente para oprimir a la mujer, y que la hace aparecer más primitiva, más cercana a la naturaleza que los hombres. ${ }^{78}$ Así, este detallado estudio de la primatología mostró la importancia de diferenciar entre los procesos naturales, biológicos subyacentes: una "primera naturaleza” que no puede ser jamás capturada y mostrada exactamente como es, precisamente porque una descripción de ella debe enunciarse en un lenguaje imbuido de preconcepciones culturales e ideológicas, y una "segunda naturaleza". La investigación psicológica con demasiada frecuencia pretende describir la naturaleza en su estado crudo cuando en realidad está reciclando imágenes de "segunda naturaleza", algo que se siente como profundo y permanente, pero que puede ser cuestionado y cambiado en tiempos de revolución.

\section{El feminismo fue "conocimiento situado" en contra de la psicología "objetiva"}

Los estudios de Harlow, cuyas imágenes aún se encuentran desperdigadas por los textos académicos de psicología estadounidenses, pretenden ser investigaciones neutras, imparciales y científicas; sin embargo, estudios feministas han

76 Véase Haraway (1989) para este análisis detallado de la primatología y de las conclusiones extraídas por los psicólogos.

77 Los estudios de Harlow sobre la depresión incluyeron una serie de atrocidades contra los sujetos de prueba (simios), que incluían el confinamiento solitario en jaulas que apenas permitían el movimiento por períodos de hasta un año. Tras esta experiencia las hembras, incapaces de aparear, eran impregnadas empleando lo que Harlow denominó el rape rack ("el rack violador"), o, en su peor acepción, "el atormentador" (N. del T.).

78 Véase Burman (1994) para un relato sobre cómo este trabajo funciona ideológicamente en la psicología del desarrollo. 
demostrado que se trata de formas de conocimiento situado. La respuesta del trabajo feminista, argumentó Haraway, no debería ser pretender entregar la visión verdadera "desde la perspectiva divina" de la realidad, sino que debería ella misma estar reflexivamente consciente de cómo produce conocimiento situado desde una perspectiva parcial.

Estas nociones del conocimiento situado, de perspectiva parcial, y luego de teorías de punto de vista, que argumentan que aquellos con menos poder en una sociedad verán mejor cómo trabaja el poder, han sido cruciales para la reorientación de la investigación. ${ }^{79}$ Este trabajo de investigación feminista arroja nuevas luces, no sólo sobre cómo se ve la psicología, sino sobre cómo adquirimos la consciencia de que siempre la observamos desde un cierto punto de vista. Luego pone en tela de juicio lo que la psicología nos dice que deberíamos ser, muestra cómo lo que somos está íntimamente ligado a formas de conocimiento sobre la mente, que están construidas desde una perspectiva ajena y hostil al entendimiento creativo colectivo humano y al cambio social.

\section{[El feminismo] derivó en propuestas de compromiso socialista- feminista con la tecnología}

Un examen minucioso de las horrorosas tecnologías de control en estudios sobre primates no derivó en un rechazo de la tecnología. La lección de la primatología y de las imágenes de naturaleza cruda que algunos psicólogos afirmaban haber descubierto, cuando lo que hacían realmente era reconstruir nuestra "segunda naturaleza", fue precisamente que no sería una solución intentar "salirse" de la tecnología. La alienación no es una función de la tecnología como tal, aunque es tentador echarle la culpa al sentido de nosotros mismos como máquinas a la existencia de la tecnología. Siempre hay algún tipo u otro de tecnología y la pregunta es cómo nos posicionamos en relación a ella y en relación a los demás. ${ }^{80}$

En vez de referirse a la naturaleza real como una alternativa fantasiosa y romántica a la tecnología, los social-feministas necesitaban entender cómo particulares tipos de tecnología crean particulares tipos de subjetividad. Para combatir la tecnofobia experimentada por muchas mujeres frente a la tecnología -temor que una vez más las hace aparentar más primitivas y más cercanas a la naturaleza- se hizo evidente que nuevas formas de subjetividad distribuida pudiesen ser

79 Véase Hartsock (1987).para un argumento materialista histórico feminista a favor de la importancia del "punto de vista" en la investigación y en la acción política.

80 Gordo López y Cleminson (2004) entregan un recuento de cómo distintas prácticas tecnológicas en el curso de la historia han producido distintas formas de subjetividad y relaciones sexuales. 
un poderoso recurso para la mujer. ${ }^{81}$ Esta "segunda ola" socialista-feminista del feminismo se volvió un recurso para los argumentos feministas de "tercera ola" que nos llevan más allá de la psicología, hacia la "ciber-psicología", y, luego, más allá de esta última también. ${ }^{82}$

\section{Los vínculos de "tercera ola" con subjetividades distribuidas en la política ciberfeminista y queer ${ }^{83}$}

El manifiesto cyborg de Haraway fue una intervención en el feminismo y en el nuevo reino del ciberespacio ${ }^{84}$. Si bien pudiese ser cierto que el ciberespacio funcione como una "zona de fantasía donde el deseo masculino de trascender al cuerpo físico alcance su cenit"s5, es una tecnología contradictoria que abre espacios para el movimiento, así como también para nuevas formas de vigilancia y control. Propuestas a favor de un nuevo ciberfeminismo se unen a emergentes feminismos de "tercera ola", que son deliberada y explícitamente plurales, y que atienden a divisiones entre las mujeres, construidas alrededor de las categorías de raza y sexualidad. Tal como el marxismo ha tenido que aprender que el antirracismo y el feminismo no son distracciones de la lucha de clases, sino que son cruciales para la confianza y autoactividad de la clase trabajadora, asimismo las feministas han aprendido que el feminismo en sí debe ser una política pluralista. Este pluralismo es muy distinto, sin embargo, del pluralismo liberal que nos dice que los derechos de cada individuo alienado deben ser respetados, y se inspira en una noción diferente de derechos, más relacional y que anticipa nuevas formas de relaciones de producción y de reproducción no alienantes.

Estos feminismos abarcan la política queer, que cuestiona la división cultural de las sexualidades "normal" y "anormal", y que son flexibles, tanto en sus intervenciones políticas como en sus teorías. ${ }^{86}$ Diametralmente opuesta a la predicción y el control psicológico, una de las consignas de estos nuevos feminismos es la precariedad, un intento por entender y reclamar la naturaleza precaria de la vida e identidad bajo el capitalismo. ${ }^{87}$

\footnotetext{
Véase Haraway (1991) para una discusión de estos temas.

Véase Gordo-López y Parker (1999) para una compilación que trata estos temas.

Extraño, inusual. El término queer se usa también para referirse a la cultura homosexual. ( $\mathrm{N}$ del T.)

84 El manifiesto de Haraway (1991) también se encuentra disponible en línea (por supuesto).

85 Marsden (1999), pág. 73.

86 Para una respuesta psicológica feminista más escéptica a la teoría queer véase Kitzinger y Wilkinson (1994).

87 Precarias a la Deriva (2005) reseña esta perspectiva feminista sobre los viajes psicogeográficos perturbadores por locaciones cotidianas.
} 


\section{Un nuevo enfoque sobre la conciencia surgió en América Latina en los años 1980}

La Unión Soviética se encontraba lo suficientemente lejos de Estados Unidos para funcionar como una pantalla sobre la cual proyectar toda clase de conspiraciones y amenazas; para algunos era un imperio del mal que servía para confirmar que una alternativa socialista al capitalismo debía ser una brutal dictadura. Lamentablemente, para otros, parecía el lugar donde por fin se había realizado el paraíso en la tierra. Las revoluciones en Latinoamérica, que a Estados Unidos le gustaba pensar que era su propio patio trasero, significaban una amenaza más directa. Lo peor de la revolución cubana de 1959 para la clase dominante, y más aún, de la de Nicaragua en $1979,{ }^{88}$ fue que ambas revoluciones eran más genuinamente democráticas que los regímenes apoyados por Estados Unidos que dichas revoluciones derrocaron. Los sandinistas en Nicaragua incorporaron sacerdotes jesuitas entre sus dirigentes, y la teología de la liberación fue incluida en la agenda como una fuerza movilizadora que cambiaría el concepto que tenía la gente de la espiritualidad puesta en práctica. Mientras Marx había tratado la religión como el opio de los pueblos o -más generosamente- "el corazón de un mundo sin corazón", la teología de la liberación mostró que las concepciones religiosas del sí mismo y de la realidad podían llevar a un compromiso de cambiar el mundo. ${ }^{89}$ Los psicólogos, que gustan de explicar las ideas religiosas como ilusiones, en el mejor de los casos, tratándose de los neuróticos por ejemplo, y en el peor de los casos, como sintomáticas de la psicosis, también estaban destinados a recibir un shock. La nueva generación de sacerdotes-obreros tenía algunas enseñanzas para las concepciones psicológicas de la salud mental que mostraban que la revolución podía ser buena para uno. Los efectos de las sanciones contra Cuba, la caída del Muro de Berlín en 1989, y la consecuente pérdida de apoyo económico, sumado al éxito de los Contras en Nicaragua, apoyados por Estados Unidos, aumentaron la presión sobre Castro. Así como el "socialismo en un solo país" era imposible en la Unión Soviética, el yugo impuesto por el imperialismo sobre Cuba ha derivado en un grado de burocratización del régimen, sin perjuicio de que los logros de la revolución merecen ser defendidos ${ }^{90}$. El mundo desalmado se está entrometiendo en los asuntos de Latinoamérica con más brutalidad que nunca.

88 Para una discusión favorable de los servicios de salud mental en Nicaragua tras diez años de revolución véase Harris y Shefer (1990); para un relato más bien rosa sobre el sistema de salud mental cubano, Michie (1979).

89 Existe una discusión marxista de la teología de la liberación y sus implicancias para la política revolucionaria en Löwy (1988).

90 La pregunta, por supuesto, es si uno "defiende" una revolución silenciando los abusos de poder, en cuyo caso uno termina meramente defendiendo a la jerarquía del aparato partidario; 


\section{Martín-Baró vinculó la interpretación del mundo con el cambio, a fin de combatir las mentiras}

Ignacio Martín-Baró, para citar un caso significativo, era un sacerdoteobrero originario del País Vasco, pero en ese entonces radicado en El Salvador, que tenía además formación como psicólogo, aunque entendía la psicología como algo que fue impuesto mediante el proceso de la colonización. Abordar la conducta como una cosa que podía condicionarse premiándola con refuerzo positivo, como proponían los conductistas estadounidenses, era perder de vista la crucial capacidad de los seres humanos de ser agentes reflexivos. Lo que sabemos sobre el mundo afecta lo que es posible hacer en él.

Para Martín-Baró, un cierto tipo de mentalidad del colonizado necesitaba ser abordado como parte del proceso de cambio. No era posible tratar los problemas psicológicos con cualquier aproximación "objetiva”, sino que era necesario entablar un compromiso subjetivo de des-ideologización a fin de devolver a la gente el conocimiento que se le había robado. Este trabajo se volvió crecientemente influyente durante la década de los ochenta en Latinoamérica, desde México, pasando por Centroamérica, hasta el sur del continente.

\section{La elección de ciertas preguntas y el uso táctico de los métodos psicológicos llevaron a su asesinato}

Una vez adoptado el enfoque de Martín-Baró, uno comienza a ver que "el rol de los sujetos experimentales es aquel de objetos idiotas". ${ }^{91}$ Sin embargo, a veces era necesario utilizar tácticamente las herramientas de sondeo de actitudes desarrolladas por las corrientes psicológicas tradicionales. Martín-Baró insistía que los proyectos políticos del investigador que trabaja junto con los oprimidos debían definir cuáles herramientas se utilizan y cómo se interpretan. Hemos visto cómo los métodos cualitativos en la psicología no son necesariamente más progresistas que las investigaciones cuantitativas estándar, y que existen muchos estadísticos radicales que tienen mucho interés de utilizar conocimientos científicos detallados sobre la naturaleza de la inequidad social a fin de apoyar a los movimientos de cambio social. ${ }^{92}$

Martín-Baró, por ejemplo, llevó a cabo encuestas de opinión a través de de la frontera de Nicaragua que demostraron, en contra de las mentiras que propagaban los terroristas contras apoyados por Estados Unidos, que el régimen sandi-

o si uno defiende el propio proceso revolucionario al oponerse a la intervención imperialista al mismo tiempo de apoyar a las revolucionarias voces democráticas de la disidencia.

Jiménez-Domínguez (2005), página 66.

92 Para colecciones del Radical Statistics Group o Grupo de Estadísticos Radicales, véase Irvine et al. (1979) y Dorling y Simpson (1999). 
nista gozaba aún del apoyo de la mayoría de la población. Martín-Baró realizaba encuestas parecidas en El Salvador, en 1989, cuando un escuadrón de la muerte, de la dictadura militar, irrumpió en la Universidad Centroamericana donde él trabajaba y lo acribilló junto a colegas sacerdotes y empleados domésticos.

\section{El cuestionamiento de lo dado por sentado fue vinculado a la concientización}

La tarea de la educación es transformada en el marco de la teología de la liberación propuesto por Martín-Baró. Como los marxistas siempre han discutido, es necesario educar al educador, en vez de dar por sentado que los revolucionarios poseen una experticia que está por encima de lo que para las personas comunes es posible de comprender. El proceso de concientización en que Martín-Baró se embarcó lo llevó a desarrollarse más allá de la teología de la liberación, hasta llegar a la perspectiva hoy conocida como la psicología de la liberación. ${ }^{93}$

Los psicólogos occidentales suponen que ya saben cómo es el mundo y lo que es posible que uno sea en él; mientras tanto, en Latinoamérica, Martín-Baró y otros investigadores de la acción participativa, se autoadjudicaron la tarea de trabajar con gente que empezaba a cuestionar lo que era posible. Esta tradición de trabajo incluye a Pablo Freire en Brasil, Orlando Fals Borda en Colombia y Maritza Montero en Venezuela, y cambia el lugar hacia donde dirigimos la mirada como psicólogos, para encontrar el verdadero material de la psicología. Las críticas formuladas sobre esta perspectiva en Latinoamérica, sobre que existe el riesgo de que uno simplemente se sumerja en una comunidad, perdiendo toda distancia crítica del sentido común cotidiano, se han desarrollado en solidaridad con el enfoque global $^{94}$. Estas críticas indican que el campo en donde emerge la conciencia en la psicología de la liberación es un campo de perspectivas diversas, conflictivo, dialéctico, y con raíces en la práctica.

\section{Desde los "centros" a los "márgenes"}

La psicología de la liberación es muy distinta a la psicología que se ha desarrollado en los sobredesarrollados centros de mundo, y nos muestra que esta última forma de psicología reflejará ella misma aquel lugar de origen en la forma que adopte. Existe la suposición, en la psicología occidental, que la psicología individual debe tener un centro - un punto de funcionamiento mental integrado al interior de cada persona- y tal suposición es una función del viejo supuesto co-

93 Véase Martín-Baró (1994) para una colección de escritos sobre la psicología de la liberación.

94 Para una crítica simpatizante con el proyecto global véase Jiménez-Domínguez (1996). 
lonial acerca de que los sistemas económicos y políticos sólo pueden funcionar sin contratiempos si hay centros que los guíen. Esta presunción luego refuerza el individualismo de la psicología occidental, y formas de psicologización han operado haciendo que cada persona sienta que tiene un poco de psicología dentro de ella.

Nuevas formas de globalización y neoliberalismo han transformado esas presunciones sobre centros y márgenes, pero necesitamos entender cómo las nuevas formas de control "distribuido" aún perpetúan sistemas de producción económica y de consumo en que cada trabajador, individual y consumidor, no tiene poder alguno para determinar qué sucederá con el fruto de su trabajo. La psicología de la liberación es, de hecho, "el suspiro de una criatura oprimida", y obliga a los psicólogos en occidente a examinar la naturaleza de la opresión y verla como amarrada al control colonial y al racismo neocolonial.

\section{La resistencia es necesaria y posible en la psicología y en contra de ella}

La Revolución Rusa, las revueltas estudiantiles en Francia, el feminismo estadounidense y los movimientos de liberación latinoamericanos, remecieron y perturbaron los supuestos que los psicólogos habían formulado sobre el ser humano. La naturaleza de la psicología como una disciplina reduccionista e individualizante fue puesta en tela de juicio y se abrieron distintos puntos de vista sobre la naturaleza de la alienación. Más importante aún, las maneras de entender lo que era la alienación -y cómo la explotación económica se intersectaba con las formas de opresión sexual, de género o cultural- estaban íntimamente ligadas al cambio. Una relación dialéctica entre la interpretación y el cambio fue forjada en esos momentos revolucionarios.

\section{Es necesario entender el peso del pasado y lo que ha sido posible}

Es igualmente importante saber cómo estas posibles nuevas aproximaciones a la psicología se han clausurado, así como el cómo emergieron. El cierre alrededor de una imagen fija y limitada del individuo hace que los distintos tipos de trabajo vuelvan a ser nuevamente compatibles con las corrientes principales de la psicología.

La traducción del trabajo de Vygotsky al inglés, por ejemplo, ha permitido que algunos psicólogos occidentales radicales se informen sobre las posibles características de la psicología revolucionaria, pero su trabajo también ha sido absorbido de vuelta en las corrientes principales de la psicología del desarrollo, como si se tratara de otra teoría más sobre cómo los niños se transforman en adultos. Los trabajos de Sève, de Holzkamp y el de Foucault, aún operan como puntos de referencia para la resistencia a la psicología occidental, pero, también, han sido 
despolitizados, y no existe razón alguna para que la disciplina de la psicología pudiera adoptarlos y utilizarlos para sus propios fines. Las perspectivas feministas a menudo siguen siendo muy perturbadoras para los psicólogos, ya que sacan a colación los temas de la política y la subjetividad, aunque ya existen carreras lucrativas en los ámbitos de la psicología de la mujer y la psicología lesbiana y homosexual en Estados Unidos, que han convertido el trabajo radical en especialidades académicas. Incluso el trabajo de Martín-Baró, que demuestra cómo la genuina comprensión de la naturaleza de la opresión sólo puede desarrollarse mediante la revolución, se puede formalizar e incorporar a la psicología comunitaria. No hay garantía de que las ideas que alguna vez fueron revolucionarias permanezcan radicales por siempre, pero existe el potencial para ello, potencial que depende de que nosotros mantengamos vivas las historias alternativas que apuntan hacia la emancipación.

\section{Trabajamos desde las grietas de las actuales relaciones de poder con el propósito de abrir paso a algo nuevo}

Las ondas expansivas revolucionarias cambiaron la visión que los psicólogos tenían del mundo, y las estelas de sus enseñanzas siguen emanando desde sus puntos de origen: el "trabajo de memoria" de Haug ha sido rescatado y desarrollado por psicólogos feministas alrededor del mundo, en estudios críticos de la psicología educacional en Australia, por ejemplo. ${ }^{95}$ El trabajo de Foucault sobre el poder se ha utilizado en los análisis sobre la nueva psicología de la paz en Sudáfrica. ${ }^{96}$ Las luchas móviles y precarias, inspiradas en parte por el trabajo de Haraway, han sido empleadas por psicologías feministas alrededor del mundo; ${ }^{97}$ la investigación-acción-participante se ha difundido al mundo imperialista, para educar a los educadores. ${ }^{98}$

Las revoluciones remecieron a la psicología e introdujeron nuevos conceptos en la agenda: actividad, poder, "segunda naturaleza", y conciencia colectiva. Pero la pregunta ahora es cómo vinculamos estos conceptos entre sí. En vez de

95 Véase Davies y Gannon (2006). Hay una buena discusión de los problemas conceptuales en el desarrollo del "trabajo de memoria" por Stephenson (2003).

96 Durkheim (1997) analiza las funciones de la retórica sobre la "paz" durante el período de transición en que fue desmantelado el apartheid. Este escrito es uno de una serie de estudios de "análisis de discurso" que señala que el contexto político en Sudáfrica podía producir algo mucho más crítico que la "psicología discursiva" británica; para otros ejemplos véase Levett et al. (1997); y en relación al trabajo crítico en la psicología en Sudáfrica, véase Hook (2004).

97 Zavos et al. (2005) entrega una visión global y una ampliación del trabajo feminista y activista en, y en contra de la psicología.

98 Véase Collins (2003) para un ejemplo de la "investigación acción” sustentada por la política revolucionaria. 
simplemente repetir los descubrimientos logrados durante esos tiempos revolucionarios, descubrimientos enraizados en sus particulares contextos, necesitamos saber cómo aprender de ellos para poder realizar nosotros algo revolucionario en, y en contra de, la psicología en estos tiempos.

\section{Bibliografía}

Bickley, R. (1977) Vygotsky's contributions to a dialectical materialist psychology, Science \& Society, 41, pp. 191-207.

Blackman, L. (1994) What is doing history? The use of history to understand the constitution of contemporary psychological objects, Theory \& Psychology, 4 (4), pp. 485-504.

Bratsis, P. (2006) Everyday Life and the State. Boulder, CO: Paradigm.

Braverman, H. (1976) Labor and Monopoly Capital: The Degradation of Work in the Twentieth Century. New York: Monthly Review Press.

Burman, E. (1994; edición corregida y aumentada en prensa) Deconstructing Developmental Psychology. London and New York: Routledge.

Burman, E. (1995) The abnormal distribution of development: Policies for southern women and children, Gender, Place and Culture, 2 (1), pp. 2136.

Burman, E. (1997) Telling stories: Psychologists, children and the production of "false memories", Theory \& Psychology, 7 (3), pp. 291-309.

Burman, E. (2004) Taking women's voices: The psychological politics of feminisation, Psychology of Women Section Review, 6 (1), pp. 3-21.

Burman, E. (2005) Engendering culture in psychology, Theory \& Psychology, 15 (4), pp. 527-48.

Burman, E. (2006) Emotions and reflexivity in feminised action research, Educational Action Research, 14 (3), pp. 315-32.

Burman, E. (in press) Between orientalism and normalization: Lessons from Japan for developmental psychology', History of Psychology.

Burman, E. and Chantler, K. (2002) Service responses to South Asian women who attempt suicide or self harm: Challenges for service commissioning and delivery. Critical Social Policy, 22 (4), pp. 641-68.

Burman, E., Aitken, G., Alldred, P. Allwood, R., Billington, T., Goldberg, B., Gordo-López, Á. J., Heenan, C., Marks, D. y Warner, S. (1996) Psychology Discourse Practice: From Regulation to Resistance. London: Taylor \& Francis.

Colletti, L. (1970) The question of Stalin, New Left Review, 61, pp. 61-81.

Collier, A. (1994) Critical Realism: An Introduction to Roy Bhaskar's Philosophy. London: Verso.

Collins, C. (2003) Critical psychology and contemporary struggles against neoliberalism, Annual Review of Critical Psychology, 3, pp. 26-48.

Curt, B. C. (1994) Textuality and Tectonics: Troubling Social and Psychological Science. Buckingham: Open University Press. 
Davies, B. and Gannon, S. (2006) Doing Collective Biography. Buckingham: Open University Press.

Debord, G. (1977) Society of the Spectacle. Detroit: Black and Red.

Deleuze, G. and Guattari, F. (1977) Anti-Oedipus: Capitalism and Schizophrenia. New York: Viking.

Dorling, D. And Simpson, S. (eds) (1999) Statistics in Society: The Alithmetic of Politics. London: Edward Arnold.

Durrheim, K. (1997) Peace talk and violence: An analysis of the power of "peace", in A. Levett, A. Kottler, E. Burman and I. Parker (eds) Culture, Power and Difference. Discourse Analysis in South Africa. London: Zed.

Eisenstein, H. (1996) Inside Agitators: Australian Femocrats and the State. Philadelphia, PA: Temple University Press.

Fanon, E (1967) The Wretched of the Earth. Harmondsworth: Penguin.

Fanon, E (1970) Black Skin White Masks: The Experiences of a Black Man in a White World. London: Paladin.

Foucault, M. (1977) Discipline and Punish: The Birth of the Prison. Harmondsworth: Penguin.

Foucault, M. (1991) Remarks on Marx: Conversations with Duccio Trombadori. New York: Semiotext(e).

Friedman, D. (1990) The Soviet Union in the 1920s: An historical laboratory, Practica, 7 (3), pp. 5-8.

Gamble, S. (2001) The Routledge Critical Dictionary of Feminism and Postfeminism. London and New York: Routledge.

Gambles, R., Lewis, S. And Rapoport, R. (2006) The Myth of Work-Life Balance: The Challenge of Our Time for Men, Women and Societies. Chichester: Wiley.

Geras, N. (1983) Marx and Human Nature. London: Verso.

Gordo López, Á. J. And Cleminson, R. (2004) Techno-Sexual Landscapes:

Changing Relations Between Technology and Sexuality. London: Free Association Books.

Gordo López, Á. J. And Parker, I. (eds) (1999) Cyberpsychology. London: Palgrave.

Gordo López, Á. J. y Linaza, J. L. (comes) (1996) Psicologías, Discursos y Poder (PDP). Madrid: Visor.

Greenslade, L. (1996) V. N. Volosinov and social psychology, in I. Parker and R. Spears (eds) Psychology and Marxism: Coexistence and Contradiction. London: Pluto.

Haraway, D. J. (1989) Primate Visions: Gender, Race, and Natura in the World of Modern Science. London and New York: Routledge.

Haraway, D. J. (1991) Simians, Cyborgs, and Women: The Reinvention of Nature. London and New York: Routledge. A cyborg manifesto: Science, technology, and socialist-feminism in the late twentieth century, included in this book, is available at www.kitchenmedialab.org/download/cyborgmanifestol. $\mathrm{rtf}$ (accessed 24 August 2006). 
Harris, A. and Shefer, T. (1990) Mental health services in Nicaragua: Ten years of revolution, Psychiatric Bulletin, 14, pp. 346-50.

Harris, B. (1990) Psychology, in M.J. Buhle, P. Buhle and D. Georgakas (eds) Encyclopedia of the American Left. New York: Garland.

Harris, B. (1995) Psychology and Marxist politics in America, in I. Parker and R. Spears (eds) Psychology and Marxism: Coexistence and Contradiction. London: Pluto.

Hartsock, N. (1987) The feminist standpoint: Developing the ground for a specifically feminist historical materialism, in S. Harding (ed.) Feminism and Methodology. Social Science Issues. Bloomington, IN: Indiana University Press.

Haug, F. (1987) Female Sexualisation. London: Verso.

Haug, F. (2000) 'Memory work: The key to women's anxiety', in S. Radstone (ed.) Memory and Methodology. Oxford: Berg.

Hayes, G. (1996) The psychology of everyday lile, in I. Parker and R. Spears (eds) Psychology and Society. Radical Theory and Practice. London: Pluto.

Hayes, G. (1998) We Buffer our memories: Thinking about the past, healing and reconciliation, American Imago, 55, pp. 29-50.

Heartfield, J. (2002) The Death of the Subject Explained. Sheffield: Perpetuity.

Heather, N. (1976) Radical Perspectives in Psychology. London: Methuen.

Hedges, L. V. (1987) How hard is hard science, how soft is soft science?, American Psychologist, 42, pp. 443-55.

Held, J. (2006) Beyond the mainstream: Approaches to critical psychology in the German-speaking community and their International significance, $A n$ nual Review of critical Psychology, 5, available at www.discourseunit. com/ arcp/S.htm (accessed 13 September 2006).

Hoggett, P. (1996) Emotion and politics', in I. Parker and R. Spears (eds) Psychology and Society: Radical Theory and Practice. London: Pluto.

Holloway, J. (2002) Change the World without Taking Power. The Meaning of Revolution Today. London: Pluto.

Holzkamp, K. (1992) On doing psychology critically, Theory \& Psychology, 2 (2), pp. 193-204.

Holzman, L. (1996) Newman's practice of method completes Vygotsky, in I. Parker and R. Spears (eds) Psychology and Marxism: Coexistence and Contradiction. London: Pluto.

Holzman, L. (ed.) (2006) What kind of theory is activity theory? Special Issue', Theory \& Psychology, 16 (1).

Holzman, L. And Mendez, R. (2003) Psychological Investigations: A Clinícian1s Guide to Social Therapy. New York and Hove: Brunner-Routledge.

Hood, L. And Newman, F. (1983) Tools and results: Understanding, explaining and meaning (three sides of one dialectical coin), Practice, 1 (2/3), pp. 154-88.

Hook, D. (ed.) (2004) Critical Psychology. Cape Town: University of Cape Town Press. 
Ingleby, D. (1972) Ideology and the human sciences: Some comments on the role of reification in psychology and psychiatry, in T. Pateman (ed.)Counter Course: A Handbook for Course Criticism. Harmondsworth: Penguin.

Irvine, J., Miles, I. And Evans, J. (eds) (1979) Demystifying Social Statistics. London: Pluto.

Jiménez-Domínguez, B. (1996) Participant Action Research: Myths and fallacies, in I. Parker and R. Spears (eds) Psychology and Society: Radical Theory and Practice. London: Pluto.

Jiménez-Domínguez, B. (2005) The critical and liberationist social psychology of Ignacio Martín-Baró: An objection to objectivism, Journal of Critical Psychology, Counselling and Psychotherapy, 5 (2), pp. 63-9.

Johnson, R. D. And Downing, L. L. (1979) Deindividuation and valence of cues: Effects on prosocial and antisocial behaviour, Journal of Personality and Social Psychology, 37, pp. 1532-8.

Kitzinger, C. and Wilkinson, S. (1994) Virgins and queers: Rehabilitating heterosexuality?, Gender \& Society, 8 (3), pp. 444-63.

Kozulin, A. (1989) Vygotsky's Psychology. A Biography of Ideas. Hemel Hempstead: Harvester Wheatsheaf.

Kozulin, A. (1994) Psychology in Utopia: Toward a Social History of Soviet Psychology. Cambridge, MA: MIT Press.

Laclau, E. and Mouffe, C. (2001) Hegemony and Socialist Strategy. Towards a Radical Democratic Politics, Second Edition. London: Verso.

Le Bon, G. (1896) The Crowd: A Study of the Popular Mind. London: Ernest Benn.

LeOnard, P. (1984) Personality and Ideology: Towards a Materialist Understanding of the Individual. London: Macmillan.

Levett, A., Kottler, A., Burman, E. and Parker, I. (eds) (1997) Culture, Power and Difference. Discourse Analysis in South Africa. London: Zed.

Lówy, M. (1988) Marxism and Liberation Theology. Amsterdam: IIRE.

Macey, D. (1994) The Lives of Michel Foucault. London: Vintage.

Macpherson, C. B. (1964) The Political Theory of Possessive Individualism. Oxford: Clarendon.

Maiers, W. and Tolman, C. (1996) Critical psychology as subject-science, in I. Parker and R. Spears (eds) Psychology and Marxism: Coexistence and Contradiction. London: Pluto.

Mandel, E. (1978) From Stalinism to Eurocommunism: The Bitter Fruits of

'Socialism in One Country'. London: New Left Books.

Marsden, J. (1999) Cyberpsychosis: The feminization of the post-biological body, in Á. J. Gordo López and I. Parker (eds) Cyberpsychology. London: Palgrave.

Martín-Baró, I. (1994) Writings for a Liberation Psychology. Cambridge, MA: Harvard University Press.

10 August 2006). 
McDougall, W. (1927) The Group Mind (originally published 1920). New York: G. P. Putnam.

Michie, S. (1979) The psychiatric hospital in Havana, Bulletin of the British Psychological Society, 32, pp. 143-4.

O’ Donnell, P. (1982), Lucien Sève, Althusser and the contradictions of the PCF, Critique, 15, pp. 7-29.

Parker, I. (1995b) Michel Foucault, psychologist, The Psychologist, 8 (11), pp. 214-16.

Plant, S. (1993) The Most Radical Gesture: The Situationist International in a Postmodern Age. London: Routledge.

Rоwвотнам, S. (1973) Hidden from History: 300 Years of Women's Oppression and the Fight Against It. London: Pluto.

SAnin, D. (2006) Critical psychology in Austria, Annual Review of Critical Psychology, 5, available at www.discourseunit.com/arcp/S.htm (accessed 13 September 2006).

Sawacki, J. (1991) Disciplining Foucault. Feminism, Power and the Body. London: Routledge.

SÉve, L. (1978) Man in Marxist Theory, and the Psychology of Personality. Hassocks: Harvester Press.

Shames, C. (1981) The Scientific Humanism of Lucien Séve, Science and Society, 45 (1), pp. 1-23.

Sharp, P. (2001) Nurturing Emotional Literacy: A Practical Guide for Teachers, Parents and Those in the Caring Professions. London: David Fulton.

Stephenson, N. (2003) Rethinking collectivity: Practising memory-work, International Journal of Critical Psychology, 8, pp. 160-76.

Stephenson, N. and Papadopoulos, D. (2006) Analysing Everyday Experience:

Social Research and Political Change. London: Palgrave Macmillan.

Tolman, C. (1994) Psychology, Society and Subjectivity: An Introduction to German Critical Psychology. London and New York: Routledge.

Tolman, C. And Maiers, W. (eds) (1991) Critical Psychology. Contributions to an Historical Science of the Subject. Cambridge: Cambridge University Press.

Totтon, N. (2000) Psychotherapy and Politics. London and Thousand Oaks, CA: Sage.

Trotsky, L. (1973) The Revolution Betrayed. What Is the Soviet Union and Where Is It Going? (originally published 1936). London: New Park.

Trotsky, L. (1977) The History of the Russian Revolution (originally published 1934). London: Pluto.

Trotter, W. (1919) Instincts of the Herd in Peace and War, Second Edition. London: Ernest Benn.

Vygotsky, L. S. (1962) Thought and Language. Cambridge, MA.: MIT Press.

Vygotsky, L. S. (1966) Development of the higher mental functions, in A. N. Leontiev, A. R. Luria and A. Smirnov (eds) Psychological Research in the USSR. Moscow: Progress Publishers. 
Walkerdine, V. (1982) From context to text: A psychosemiotic approach to abstract thought, in M. Beveridge (ed.) Children Thinking Through Language. London: Edward Arnold.

Zaretsky, E. (1976) Capitalism, the Family, and Personal Life. London: Pluto.

Zavos, A., Biglia, B., Clark, J. and Motzkau, J. (eds) (2005) Feminisms and Activisms, Special Issue, Annual Review of Critical Psychology.

Zhang, S. X. (1995) Measuring shaming in an ethnic context, British journal of Criminology, 35 (2), pp. 248-62. 